\title{
Grouping and ordering schemata in competition'
}

\author{
James L. Kuethe and Clinton B. De Soto \\ JOHNS HOPKINS UNIVERSITY
}

\begin{abstract}
Two experiments were performed to determine which is the more powerful, the grouping schema or the ordering schema, when they are placed in competition. It was found that, when free to do either, people tend to group elements rather than order them. It was also found that the two schemata are not inherently antagonistic; people will readily employ both when permitted and they can be mutually facilitory. One exceptional case occurs when people have the choice of augmenting a group or an ordering with an internal gap: filling in the ordering is more attractive than adding to the group.

\section{Problem}

It has been demonstrated recently that in various tasks in which people are required to interrelate or structure elements they are very prone to give the elements simple groupings or orderings at the expense of other varieties of organization (De Soto, 1960; 1961; De Soto \& Bosley, 1962; De Soto \& Kuethe, 1959; Kuethe, 1962; 1964). People have powerful grouping and ordering schemata.

The present experiments were aimed at answering the question, which of these powerful schemata proves the more powerful when they are placed in competition?

\section{Method}

The felt-figure technique (Kuethe, 1962) was used. Under this technique Ss are given objects cut from felt and allowed to place these objects on a felt field (to which they will cling) in any manner they choose.

In the first experiment, the objects were all rectangles, varying in size, to arouse an ordering schema, and in color (yellow and blue), to arouse a grouping schema. There were four sets of objects, as shown in Fig. 1, where they are ordered by size. The Ss in this experiment were 40 Johns Hopkins undergraduates.

In the second experiment, Ss were given the choice of adding a rectangle to an existing group of three identical rectangles or to an existing ordering of three rectangles varying in size. For one group of $100 \mathrm{Ss}$, the rectangle was of appropriate size to extend the ordering at one end. For another group of $100 \mathrm{Ss}$, it was of appropriate size to fill a gap in the existing ordering. For both groups of Ss, it was of the same size as the rectangles in the existing group.

\section{Results}

First experiment: Set 1 permits concordant application of a grouping and ordering schema. It was ordered by size (and at the same time grouped by color) by 36 of the $40 \mathrm{Ss}$. The other $4 \mathrm{Ss}$ grouped the objects by color without ordering them.

Set 2 arouses an ordering schema but provides no basis for color-grouping. It was size-ordered by $28 \mathrm{Ss}$.
The remaining Ss showed idiosyncratic organizations.

Set 3 introduces competition between grouping and ordering schemata; ordering the objects by size permits grouping the blue objects but precludes grouping the yellow ones. Only 14 Ss ordered Set 3; the remaining 26 chose to group the objects completely by color.

Set 4 renders grouping and ordering altogether incompatible. In this situation, only 6 Ss ordered the objects. All the remaining $34 \mathrm{Ss}$ grouped them by color.

The above data yielded a Cochran $Q$ of 47.20 , p < .001 on $3 \mathrm{df}$.

Second experiment: Ss given the choice of augmenting a group or extending an ordering chose, 61 to 39 , to augment the group. Ss given the choice of augmenting a group or filling a gap in an ordering chose, 54 to 46 , to fill in the ordering. These data yielded a $x^{2}$ of 4.52 , $\mathrm{p}<.05$ on $1 \mathrm{df}$.

\section{Discussion}

These findings indicate that the grouping schema is prepotent over the ordering schema. According to the Gestalt Law of Pragnanz, psychological organization will always be as 'good" as prevailing conditions allow. In Gestalt terms, then, the grouping and the linear ordering are both outstandingly good figures, but the grouping is the better of the two, because when conditions permit either but not both, it is chosen.

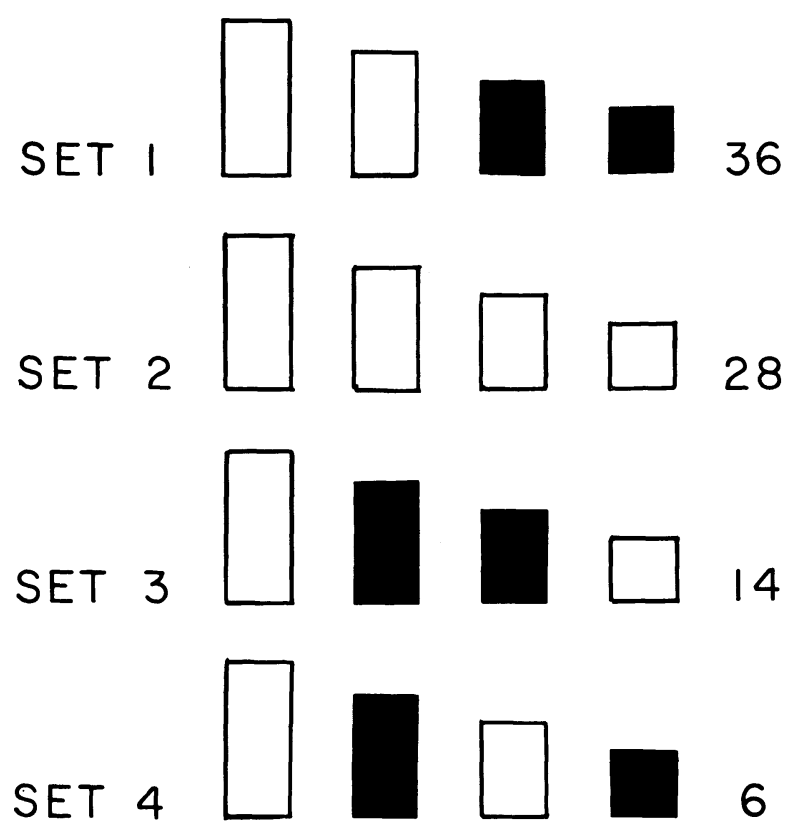

Fig. 1. Frequency with which the Ss ordered the different sets of rectangles by size. 
At the same time, the high incidence of joint sizeordering and color-grouping for Set 1 (where they could have been color-grouped without size-ordering) indicates that the two schemata are not inherently competitive or antagonistic but, on the contrary, can be mutually facilitative.

There seem also to be some forms of competition in which the tendency to order will defeat the tendency to group. For example, it has been found that people will add a disparate ordering to a grouped set of elements more readily than they will add a disparate second grouping of the elements (De Soto, 1961). The present experiments provide an additional instance in the finding that people tend to augment an ordering rather group when the ordering has an internal gap.

This last finding is reminiscent of the principles of closure and good continuation so prominent in Gestalt theory. The Gestalt psychologists, of course, applied these principles primarily to visual perception, but they argued for their applicability to cognitive processes generally, and the present experiment seems to support them in this contention. It certainly is easy to point to a wealth of anecdotal data which indicate that people strive to fill in gaps in orderings, ranging from the seductive inside straight of poker to the Catholic Church's determined historiographic efforts to keep the line of Popes unbroken to the unflagging overconcern with missing links in evolution.

\section{References}

DE SOTO, C. B. Learning a social structure.J. abn. soc. Psychol., 1960, 60, 417-421.

DE SOTO, C. B. The predilection for single orderings. J.abn. soc. Psychol., 1961, 62, 16-23.

DE SOTO, C. B., \& BOSLEY, J. J. The cognitive structure of a social structure. J.abn. soc. Psychol, 1962, 64, 303-307.

DE SOTO, C. B., \& KUETHE, J. L. Subjective probabilities of interpersonal relationships. J.abn. soc. Psychol., 1959, 59, 290-294.

KUETHE, J. L. Social schemas. J.abn. soc. Psychol., 1962, 64, 31-38.

KUETHE, J. L. Pervasive influence of social schemata. J. abn. soc. Psychol., 1964, 68, 248-254.

\section{Note}

1. This work was supported in part by Grant NSFG24025 from the National Science Foundation. 